\title{
Superradiant amplification in a chirped-tapered x-ray free-electron laser
}

\author{
J. Duris, Z. Zhang, J. MacArthur, Z. Huang, and A. Marinelli \\ SLAC National Accelerator Laboratory, Menlo Park, California 94025, USA
}

(Received 21 August 2018; revised manuscript received 6 August 2019; accepted 7 January 2020; published 6 February 2020)

\begin{abstract}
The chirp-taper free-electron laser (FEL) scheme has been proposed to generate subfemtosecond broadband pulses using laser-modulated electron bunches in x-ray FELs. In this paper, we extend the chirptaper concept to the postsaturation superradiant regime by combining an energy-modulated beam with a sinelike undulator taper. We show that subfemtosecond duration pulses with peak powers approaching a terawatt may be obtained with typical x-ray FEL parameters such as those at the Linac Coherent Light Source. The method shown here can produce a stable source of attosecond-scale, high-power x-ray pulses suitable for probing electronic dynamics within molecules.
\end{abstract}

DOI: 10.1103/PhysRevAccelBeams.23.020702

\section{INTRODUCTION}

Subfemtosecond x-ray pulses are highly desirable, as they enable the investigation of electron dynamics within atoms, molecules, and solids by probing samples faster than typical valence electron evolution timescales [1]. High-harmonic generation in gas jets is capable of producing attosecond pulses $[2,3]$, yet the produced pulse energies are typically limited to nanojoules for extreme ultraviolet and picojoules for $\mathrm{x}$ rays. X-ray free-electron lasers (FELs) offer unique advantages of tunable wavelengths and narrow bandwidths and have been shown to produce pulses as short as a few femtoseconds with peak powers of tens of gigawatts by shaping the phase space of the electron bunch at the femtosecond level [4-11]. Laser manipulation of electron beams has the potential to further reduce these pulse durations [12]. For example, enhanced self-amplified spontaneous emission (ESASE) has been proposed to produce subfemtosecond radiation pulses from femtosecond high-current electron bunches made by modulating the electrons' energy on an optical scale and then compressing via a chicane [13-16]. Combining a nonlinear electron beam energy chirp with a linear taper has been suggested to further reduce the pulse duration $[17,18]$. Power enhancement beyond the saturation level has been demonstrated by cascading amplification with a fresh-slice scheme [19,20], and a combination of slicing and cascading has been proposed to generate attosecond pulses with terawatt-level peak powers [15,21-23].

Published by the American Physical Society under the terms of the Creative Commons Attribution 4.0 International license. Further distribution of this work must maintain attribution to the author(s) and the published article's title, journal citation, and DOI.
In this paper, we propose a simple scheme to generate terawatt-level subfemtosecond pulses by driving a chirptapered free-electron laser in the postsaturation superradiant regime. In an FEL, a high-brightness relativistic electron beam travels in a magnetic undulator, generating high-power radiation pulses by means of a collective instability [24,25]. The central wavelength is defined by the resonance condition

$$
\lambda_{r}=\frac{\lambda_{u}}{2 \gamma^{2}}\left(1+K^{2}\right)
$$

where $\lambda_{r}$ is the emission wavelength (also referred to as resonance wavelength), $\lambda_{u}$ is the undulator period, $K$ is the rms undulator parameter, and $\gamma$ is the beam's Lorentz factor. FELs typically operate with a flat energy phase space so that the entire beam is resonant around the same wavelength. If the electron beam has an energy chirp, the FEL instability can be preserved by properly tapering the undulator so that the radiation stays resonant with the local electron energy as it slips ahead of the beam [17]. In the linear regime, the chirp-taper matching condition is found by taking a derivative of Eq. (1) and is given by

$$
\frac{d K}{d z}=\frac{\lambda_{r}^{2}}{\lambda_{u}^{2} K} \frac{d \gamma^{2}}{d s}
$$

where $z$ is the position along the undulator and $s$ is the position along the electron bunch. Conventionally, a taper is called negative if $d K / d z>0$; however, for clarity in this paper, we note that Eq. (2) shows that $d K / d z$ and $d \gamma / d s$ have the same sign.

If the chirp is nonlinear, lasing occurs only in regions of the electron bunch where the chirp-taper matching condition is met, and sinusoidal modulation results in a train of radiation pulses, each of which are much shorter than the 


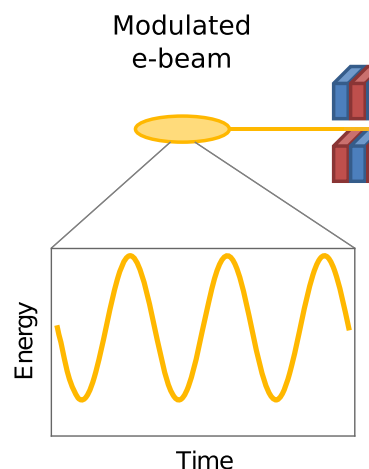

Time

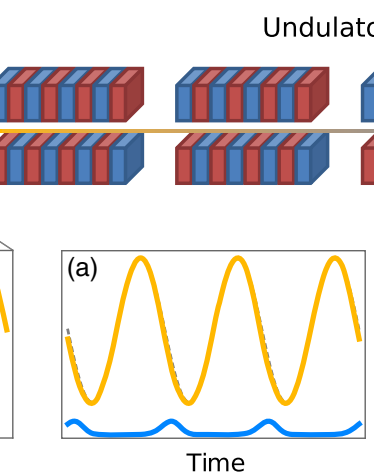

Time
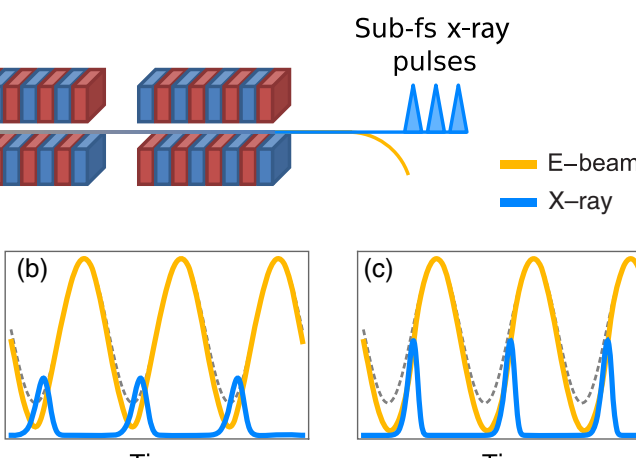

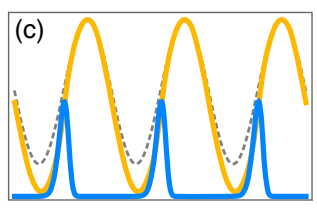

Time
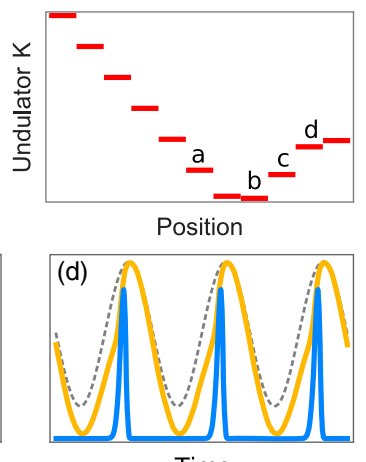

FIG. 1. Diagram of the chirp-taper setup along with a cartoon of the temporal dynamics. The bottom row of plots depicts the electron beam longitudinal phase space (yellow curves) and x-ray power (blue curves) at various points along the undulator line (labeled), depicted top right. After generating a single spike of radiation via a linear chirp-taper section, the undulators are varied to maintain resonance as the radiation advances over the electron beam.

modulation period. Simulation studies have shown that subfemtosecond x-ray pulses can be generated by modulating an electron bunch with a high-power infrared laser and employing the chirp-taper matching condition [17]. Whereas these studies have investigated chirped-tapered FELs up to the saturation point, extending the interaction by tapering the undulator to maintain resonance as the radiation slips past the initial lasing region to interact with fresh electrons leads to superradiant amplification of the initial pulse.

The superradiant regime of free-electron lasers was first discovered by Bonifacio, McNeil, and Pierini [26]. This regime occurs when a coherent radiation pulse with a duration comparable to the slippage in a synchrotron period interacts with a fresh electron bunch in an undulator. One such source of radiation may be produced by seeding an FEL with a short pulse of radiation to produce one SASE spike at saturation [27]; however, in X-ray FELs where such seed radiation sources are unavailable, a chirp-taper FEL at saturation can produce a single SASE radiation spike with a length characterized by the cooperation length, which is comparable to the slippage in half of a synchrotron period at saturation. (Here, the cooperation length is defined as the slippage in a gain length or $l_{c}=\lambda_{r} / 4 \pi \rho$, where $\rho$ is the FEL Pierce parameter.) In this strongly nonlinear regime, the head of the radiation pulse interacts with fresh electrons and extracts energy from them, and the pulse duration is determined by the slippage length acquired in a synchrotron oscillation period. The result is that the peak power increases quadratically with the interaction distance while the pulse duration decreases as $1 / \sqrt{z}$ [28]. Furthermore, the pulse duration goes as the slippage in a synchrotron oscillation period, which scales as $\lambda_{r} / z_{s} \propto \lambda_{r}^{1 / 2}$, so lasing at shorter wavelengths should further reduce the pulse duration. Consequently, driving an FEL in the superradiant regime is highly desirable for ultrafast pump-probe applications, since both the pulse duration and the peak power can be improved well beyond the standard single-spike SASE operation.
The FEL instability may limit superradiant amplification in linear chirp-taper schemes. SASE radiation pulses can form along the resonant part of the beam, spoiling the gain of the superradiant pulse by increasing the electron beam's energy spread. This competing effect can be suppressed in a sinusoidal chirp-taper setup, since the resonance condition is met only at specific locations in the electron bunch, suppressing the FEL instability everywhere else in the bunch. Consequently, extending the chirp-taper FEL to the superradiant regime makes an already femtosecond-short pulse even shorter and allows the pulse power to continue to grow.

Superradiant amplification via resonant energy slicing presented in this paper offers unique benefits compared to existing short-pulse FEL techniques. Temporal spoiling via a slotted foil, laser heater, or dechirper reduces pulse durations by removing electrons from the lasing process. ESASE compresses the beam to reduce the cooperation length in a short region of electron beam and, thus, the resulting pulse duration. In contrast, the duration of a pulse amplified by superradiance depends on the radiation intensity of the pulse, thus allowing the duration to continue to decrease as the power continues to grow. Finally, whereas fresh-bunch or fresh-slice FEL schemes can be used for pulse shortening via superradiance at a cost of skipping electrons during the delay, continuous resonant energy slicing allows all electrons the pulse passes to contribute to the lasing for greater overall power conversion efficiency.

\section{SETUP DESCRIPTION}

Our simulation study starts with the chirp-taper scheme of Saldin, Schneidmiller, and Yurkov [17], relying on a sinusoidally chirped electron beam and a linearly tapered undulator, and extends it to accommodate the sinusoidal energy profile of the electron beam. The setup is depicted in Fig. 1. 
An electron beam with an optical-scale energy modulation is employed in an x-ray FEL undulator. The electron beam energy modulation may be obtained via copropagation of the electron beam with a laser pulse in a wiggler magnet. Initially, lasing on the steepest slope of an optically modulated electron beam with a linearly tapered undulator produces a short spike of radiation as in Ref. [17]. Continuing the interaction with this linearly tapered undulator as the radiation slips forward to a region of electron beam with a nonlinear energy chirp [such as an energy trough shown in Fig. 1(b)] would break the FEL resonance and halt the interaction. On the other hand, varying the undulator field via Eq. (2) to maintain a resonant interaction between the spike and electrons facilitates superradiant amplification whereby the spike duration decreases and the power increases. The result is a train of high-power spikes, each of a duration much shorter than the wavelength of the optical modulation.

\section{NUMERICAL STUDY}

Parameters considered here for a numerical study of the setup are based on those reasonably feasible for a facility such as the Linac Coherent Light Source (LCLS) [29] or the European XFEL [30] and are summarized in Table I. We study the example of a $3.4 \mathrm{GeV}$ electron beam compressed to $4.5 \mathrm{kA}$ with an $\mathrm{rms}$ slice energy spread of

TABLE I. Simulation parameters.

\begin{tabular}{lc}
\hline \hline Electron beam & \\
\hline e-beam energy & $3.38 \mathrm{GeV}$ \\
Peak current & $4.5 \mathrm{kA}$ \\
Slice energy spread (rms) & $2.8 \mathrm{MeV}$ \\
Normalized emittance & $0.4 \mu \mathrm{m}$ \\
Average beam size & $22 \mu \mathrm{m}$ \\
Bunch length & $27 \mathrm{fs}$ \\
Bunch charge & $123 \mathrm{pC}$ \\
Energy modulator & \\
Modulation period & $2 \mu \mathrm{m}$ \\
Modulation amplitude & $17 \mathrm{MeV}$ \\
Wiggler period & $32 \mathrm{~cm}$ \\
Wiggler normalized vector potential $K$ & $33.49-34.12$ \\
Wiggler peak, on-axis magnetic field & $1.12-1.14 \mathrm{~T}$ \\
Wiggler length $L_{w}$ & $3.2 \mathrm{~m}(\mathrm{ten} \mathrm{periods})$ \\
External laser wavelength & $2 \mu \mathrm{m}$ \\
External laser power & $30 \mathrm{GW}$ \\
External laser Rayleigh range $\left(15 \% L_{w}\right)$ & $1.15 \mathrm{~m}$ \\
External laser waist position & Wiggler center \\
X-ray FEL undulators & \\
Total undulators & 30 \\
Period length & $3 \mathrm{~cm}$ \\
Normalized undulator potential $K(\mathrm{rms})$ & 2.5 \\
Resonant photon energy (wavelength) & $500 \mathrm{eV}(2.48 \mathrm{~nm})$ \\
Periods per undulator & 110 \\
Separation between undulators & $60 \mathrm{~cm}$ \\
\hline \hline & \\
\hline
\end{tabular}

2.8 MeV. Table I also shows example wiggler and externally injected laser parameters designed with equations from Ref. [31].

The laser's wavelength sets the modulation period and is chosen to be $2 \mu \mathrm{m}$ so that the linearly chirped sections of the electron beam are about a cooperation length long in order to generate one initial SASE temporal mode at saturation. The laser's peak power is $30 \mathrm{GW}$, and its transverse profile is a $\mathrm{TEM}_{00}$ Gaussian mode with a Rayleigh range of $58 \mathrm{~cm}$. This laser provides an intensity large enough to imprint an energy modulation of $17 \mathrm{MeV}$, which is several times larger than the FEL resonant energy bandwidth $(\sim 0.1 \%)$ needed to suppress simultaneous, uniform FEL lasing along the bunch. The electron beam duration is $27 \mathrm{fs}$, but its time of arrival from the linac may jitter by tens to hundreds of femtoseconds, so a pulse length of at least hundreds of femtoseconds to a few picoseconds is needed to cover the jitter for stable operation. Ho:YLF lasers operate at $2 \mu \mathrm{m}$ and are capable of producing picosecond pulses with hundreds of millijoules of pulse energy, which is more than sufficient for this application [32].

Turning our attention to the wiggler, Eq. (1) requires that its period is $32 \mathrm{~cm}$ and normalized vector potential $K$ is 33 in order to keep the peak, on-axis magnetic field a realizable $1.1 \mathrm{~T}$. The wiggler length of $3.84 \mathrm{~m}$ (12 periods) was determined by solving the no-gain FEL equations for various wiggler lengths while keeping the laser focal parameters optimal for a constant $K$ wiggler (waist centered in the wiggler and Rayleigh range $15 \%$ of the wiggler length) [31]. The ponderomotive phase displacement due to the Gaussian beam's Gouy phase shift reduces the modulation amplitude by $26 \%$ but is perfectly compensated by linearly increasing $K$ along the wiggler length from 33.49 to 34.12 by linearly reducing the wiggler gap $1.9 \mathrm{~mm}$ over its entire length.

We use x-ray FEL undulators consisting of 110 periods, each $3 \mathrm{~cm}$ long with a normalized field strength $K$ of 2.5 separated by $60 \mathrm{~cm}$ breaks consistent with the LCLS undulator design. Tapering is achieved by varying the undulator parameter $K$ of each undulator while keeping the undulator parameter $K$ uniform within each undulator module. The FEL code GENESIS 1.3 [33] is used for all $x$-ray FEL simulations presented here.

In order to design the undulator taper, we first linearly taper $K$ with Eq. (2) to match the steepest chirp of the electron beam as shown in Fig. 2(a). This selects only a small region of the beam with matching chirp to generate some initial SASE radiation without spoiling the rest of the beam, and this initial SASE pulse will then undergo superradiant growth in the following sinusoidally tapered undulator section. Choosing no initial slope may cause two pulses per modulation period by lasing on the crests and troughs, whereas targeting a zero crossing of the sinusoidal chirp selects only one region of electrons per modulation period. The modulation amplitude may not be immediately 


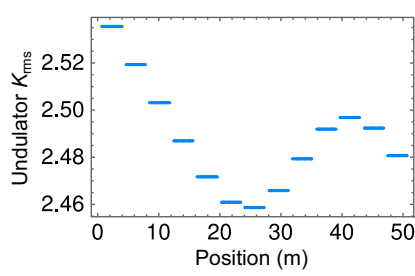

(a) Undulator taper

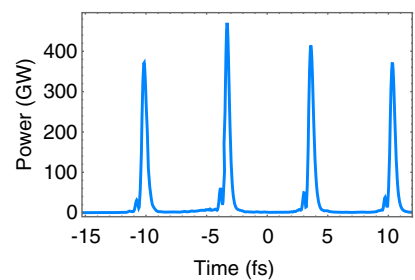

(c) Power profile at und. 10 (head is $t>0$ )

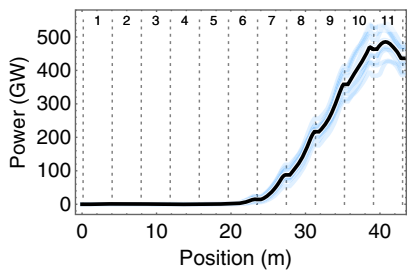

(b) Power growth

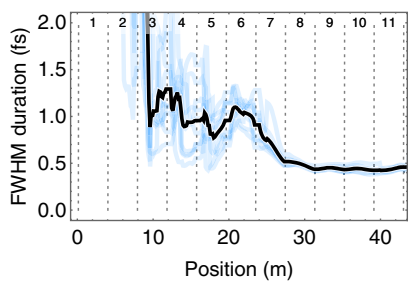

(d) FWHM pulse duration evolution
FIG. 2. Sinusoidal chirp-taper simulation results. The black lines in (b) and (d) each show the average of ten shots with different random seeds (shown in light blue). Undulator sections are demarcated by dashed vertical lines with the undulator index shown at the top of the plot's frame for reference.

measurable, so, in practice, the chirp-taper matching may be accomplished by varying the undulator taper to maximize pulse energy.

The FEL power near sections of beam with chirp matching the linear taper grows exponentially for six undulators until saturation, where the particles bunch at the FEL wavelength. Resonance is then maintained by tapering subsequent undulators so that the initial radiation spike maintains resonance with the electrons as it slips forward by one FEL period for every undulator period. Figures 2(b) and 2(d) show the pulse peak power growing and duration shrinking for undulators 7-10 in qualitative agreement with superradiant amplification. By undulator 10, the pulse peak powers exceed $400 \mathrm{GW}$, FWHM durations are less than $0.5 \mathrm{fs}$, and FWHM bandwidths are $>7 \mathrm{eV}$. Each primary pulse in the pulse train is accompanied by one or more smaller trailing pulses [see Fig. 2(c)], which is a by-product of the superradiant pulses tail churning the longitudinal phase space. This is a consequence of power in the tail accelerating electrons that were decelerated by radiation in the main pulse [34]. When the pulse has propagated through nearly a whole period of the modulation at undulator 11 , it encounters partially spoiled electrons which were previously decelerated. These decelerated electrons are detuned below resonance, thereby causing electron acceleration and removing power from the field as shown in Fig. 2(b). Continuing the interaction past this point requires accommodating for this energy change.

\section{A. Optimizing detuning}

The simulations above use a taper matching the initial energy of the electrons but ignoring radiation growth and

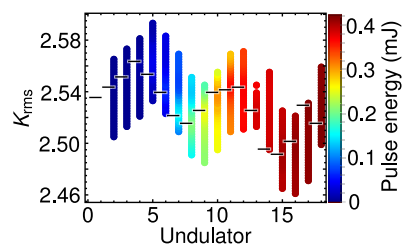

(a) Energy per pulse vs taper

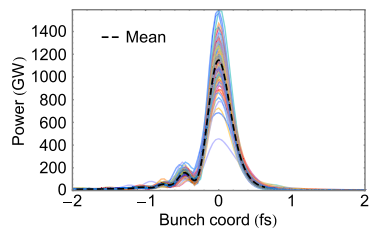

(c) Peak-aligned power profiles (head is $t>0$ )

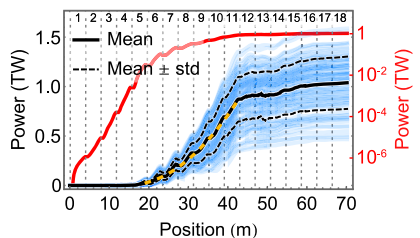

(b) Peak power along the undulator line

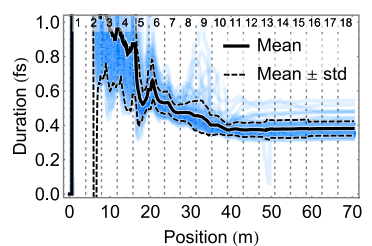

(d) FWHM duration evolution
FIG. 3. Optimal detuning chirp-taper simulations. (a) shows how undulators are varied sequentially to maximize the x-ray pulse power and energy at the output of each undulator segment (colored points) along the undulator line for an average of three shots with different random seeds. The remaining plots show the results of 100 simulations for the optimized taper shown as black line segments in (a). The dashed yellow line in (b) shows a powerlaw function fit to the mean power in undulators 5-11 with an exponent of 1.97 in agreement with superradiance, and the red line shows the log-scaled power to highlight the exponential gain in the first five undulators. (c) shows pulse profiles for the third of four pulses in the pulse train.

electron deceleration. Optimal energy extraction requires considering these effects in the taper design and results in a taper detuned from resonance [35]. In anticipation of an experimental setting where the radiation field is not readily observable, we optimize the taper numerically by varying the undulators one by one to maximize the pulse energy. A local optimization strategy suffices, since the radiation pulse is much shorter than the electron beam. Figure 3(a) shows that the taper maximizing the pulse peak power (black line segments) also maximizes the energy per pulse (colored points), yielding a method to tune up in an experimental setting where a peak power diagnostic may not be available. If the $\mathrm{x}$ rays produced in the first few undulators are insufficient for measurement, the maximum chirp may be initially estimated by the slope of a linear taper which maximizes the pulse energy. Knowledge of the modulation period allows a determination of the sinusoidal energy profile, which can then be matched by an appropriate taper to generate enough radiation to start optimizing section by section.

Figures 3(b) and 3(d) show the pulse peak power and FWHM duration along the undulators. After five undulators of exponential growth, the power grows quadratically for the next several undulators $6-11$ as the pulse undergoes superradiant amplification until it reaches electrons previously decelerated by a pulse in 


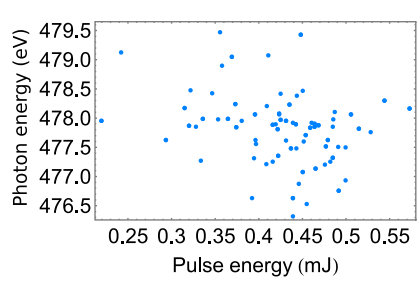

(a) Photon energy

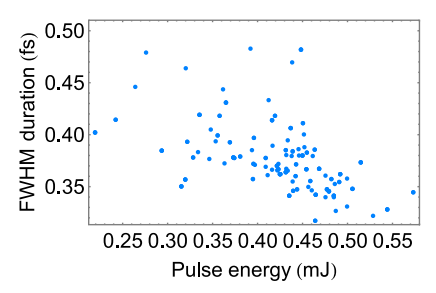

(c) FWHM duration

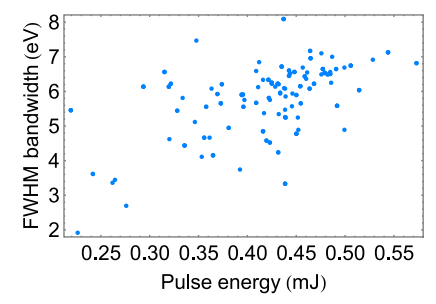

(b) FWHM bandwidth

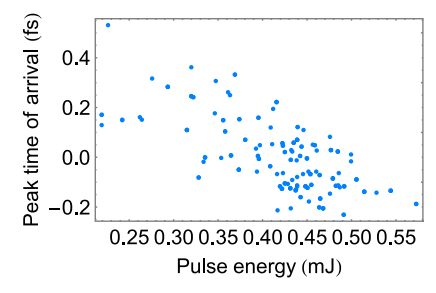

(d) Pulse peak time of arrival $(t>0$ is later $)$
FIG. 4. Distributions of shot-to-shot variations of output pulse properties after all undulators for the third of four pulses in the pulse train.

the next modulation period. This region of the beam has a larger energy spread which greatly suppresses the interaction, resulting in diminishing power extraction for the remaining undulators.

The output radiation pulses [Fig. 3(c)] have a mean peak power of $1 \mathrm{TW}$ with $25 \% \mathrm{rms}$ fluctuations from shot noise. The shot-to-shot variation of the central photon energy is $0.55 \mathrm{eV}$ [Fig. 4(a)], which is much smaller than the bandwidth, shown in Fig. 4(b). The bandwidth increases with the pulse energy and has an average of $6.3 \mathrm{eV}$ with $8 \%$ rms variation for pulses with greater than average power. The pulse duration [Fig. 4(c)] decreases with respect to the peak power as expected from superradiant amplification and has a mean of $0.36 \mathrm{fs}$ and $5 \%$ rms variation for shots with powers exceeding the mean.

The ability to determine the arrival time of $\mathrm{x}$-ray pulses is crucial for the success of time-resolved pump-probe experiments. We examined the peak positions of the output pulses [Fig. 4(d)] and observe a $0.14 \mathrm{fs}$ rms jitter-about a third of the FWHM pulse duration. These fluctuations originate from shot noise in the electron beam, so the temporal jitter of each pulse should be identical and independent, resulting in a pulse-to-pulse separation jitter of $0.2 \mathrm{fs}$ rms $(\sqrt{2}$ larger than the single pulse jitter) in agreement with double chirptaper simulations [36]. This is especially useful if one combines this tapering scheme with a two-color FEL scheme for x-ray pump, x-ray probe experiments $[9,36]$. Figure 4(d) also shows a mild correlation between the time of arrival of the peak of each pulse and its pulse energy, suggesting that time sorting may be possible for pumpprobe experiments at the subfemtosecond level. Since pulse energy is easily observable on a shot-to-shot basis, a reduction in this arrival time uncertainty (about a factor of 2 in this example) might be possible by selecting the largest pulse energies.

\section{B. Isolated attosecond pulses from self-modulated electron beams}

Isolated pulses are desirable for pump-probe experiments. While the above simulations produce pulse trains of subfemtosecond $\mathrm{x}$-ray pulses, a single pulse may be generated by using a temporally correlated beam tilt or spoiling via slotted foil to lase in a region of the electron bunch with a length similar to the modulation wavelength or by placing a single-cycle energy modulation on the electron bunch. The former method of using tilt or spoiling effectively shortens the beam by keeping electrons from lasing, whereas the latter method coupled with chirp-taper matching isolates the lasing to a short region of beam only initially, allowing for superradiant amplification with the rest of the beam. A single-cycle laser may be used to generate the required modulation, but this leads to challenges of timing stability as the electron beam at linacs such as LCLS jitters by tens to hundreds of femtoseconds [37].

On the other hand, a nearly single-cycle energy modulation has been recently demonstrated using the selfmodulation method [38,39], which relies on the coherent undulator radiation emitted by the tail of the electron bunch to modulate the electrons ahead. This method circumvents problems of spatiotemporal overlap, since the radiation is produced by the electron beam being modulated. If the chirp of the first modulation period is large enough so that the initial undulator taper matches only that one chirp, a single pulse of $\mathrm{x}$ rays may be generated and amplified along the electron bunch.

We investigate start-to-end simulations of this setup with the simulated output of the LCLS, energy modulated by self-modulation in two wigglers, compressed with a chicane, and then modulated again with two additional wigglers. The beam simulation is identical to that presented in Ref. [36] except with double the interwiggler chicane delay. The resulting longitudinal phase space, shown in Fig. 5(a), has a damped sinusoidal energy profile. The parameters for this simulation are summarized in Table II.

We initially taper five undulators to selectively lase on the steepest slope near the tail as depicted in Fig. 5(b) and then use the same procedure as before to optimize the produced pulse energy by varying the taper. Note that, when the radiation pulse interacts with the region of electron beam near $7 \mathrm{fs}$ in Fig. 5(a) where the beam energy is bimodal, competition between two local optima leads to a discontinuity in undulator $K$ variation near undulators 10 and 11. The power growth along the undulator line is shown in Fig. 5(c), where it can be seen that the power grows fastest in regions of high current $(4,11$, and 16 fs along the beam coordinate). The result is isolated pulses of radiation with peak powers reaching several hundred gigawatts and FWHM durations below 0.4 fs [Fig. 5(d)]. 


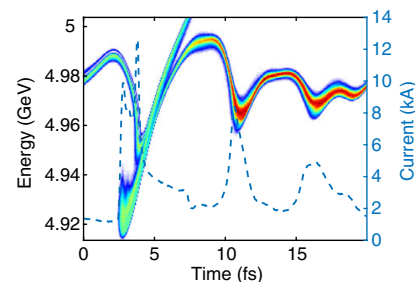

(a) Input longitudinal phase space and current (head is $t>0$ )

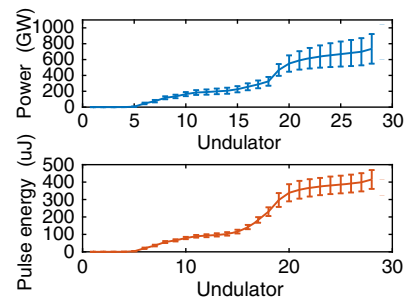

(c) Peak power and pulse energy

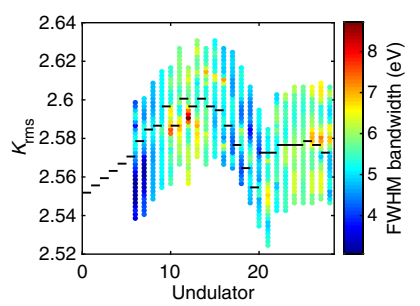

(b) FWHM bandwidth vs taper

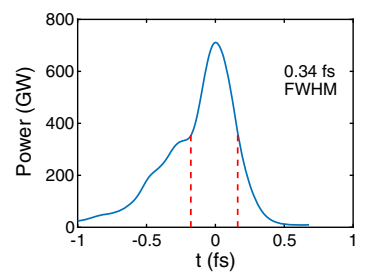

(d) Typical output pulse (head is $t>0$ )

FIG. 5. Simulation of isolated attosecond x-ray pulse generation from a self-modulated electron beam. Each simulation is repeated 10 times with different shot noise. (d) shows the power profile of a typical shot.

The simulation stops when the pulse reaches $t=15 \mathrm{fs}$ in Fig. 5(a); however, if more undulators were added, the pulse power may continue to grow as it interacts with the $5 \mathrm{kA}$ spike at $t=15$ fs. Finally, we observe from Fig. 5(b) that, by detuning the undulator slightly at a point where the radiation reaches a crest of the electron beam energy modulation, the bandwidth of the produced radiation may be increased up to 8 or $9 \mathrm{eV}$.

TABLE II. Simulation parameters.

\begin{tabular}{lc}
\hline \hline Electron beam & \\
\hline e-beam energy & $4.96 \mathrm{GeV}$ \\
Average current & $2.2 \mathrm{kA}$ \\
Slice energy spread (rms) & $3 \mathrm{MeV}$ \\
Normalized emittance & $0.4 \mu \mathrm{m}$ \\
Average beam size & $30 \mu \mathrm{m}$ \\
Modulation period & $4 \mu \mathrm{m}$ \\
Modulation amplitude & $30 \mathrm{MeV}$ \\
Bunch length & $20 \mathrm{fs}$ \\
Bunch charge & $68.5 \mathrm{pC}$ \\
Undulators & \\
Total undulators & 28 \\
Period length & $3 \mathrm{~cm}$ \\
Normalized undulator potential $K$ (rms) & 2.55 \\
Resonant photon energy (wavelength) & $1016 \mathrm{eV}(1.22 \mathrm{~nm})$ \\
Periods per undulator & 110 \\
Separation between undulators & $60 \mathrm{~cm}$ \\
\hline \hline
\end{tabular}

\section{CONCLUSION}

In conclusion, we have investigated a nonlinear chirptaper strategy to produce high-power, subfemtosecond $\mathrm{x}$-ray FEL pulses. The method produces pulse durations similar to those of existing chirp-taper schemes but extends the interaction to the superradiant regime to achieve larger peak powers and shorter-duration pulses, enabling higher contrast for subfemtosecond pump-probe experiments. Unlike traditional FEL tapering techniques, the local effect of the superradiant interaction enables tailoring the taper to unique features of the electron beam's phase space to control the pulse power and bandwidth without complications arising from the sideband instability or the need for a global taper optimization search. Furthermore, the superradiant amplification allows this scheme to achieve pulse durations shorter than a cooperation length, which is especially advantageous for soft $\mathrm{x}$-ray experiments where the slippage is significant. The pulse duration scales as $\propto \lambda_{r}^{1 / 2}$, so extending this technique to hard $\mathrm{x}$ rays should lead to even shorter pulses. This scheme is relatively simple to set up, requiring no dechirpers or beam masks, which often cause beam losses and reduced rate operation, yet can be coupled with two-color methods such as fresh slice. In this context, one can take advantage of the relatively stable 0.2 fs arrival time difference of the two pulses, enabling high-power pump-probe experiments with subfemtosecond pulses for next-generation x-ray FELs.

\section{ACKNOWLEDGMENTS}

We thank P. Baxevanis, E. Hemsing, G. Marcus, and A. A. Zholents for helpful discussions. This work was supported by the U.S. Department of Energy, Office of Basic Energy Science ADR Field Work Proposal No. 100317 as well as by the U.S. Department of Energy, Office of Science under Contract No. DE-AC0276 SF00515.

[1] F. Krausz and M. Ivanov, Attosecond physics, Rev. Mod. Phys. 81, 163 (2009).

[2] P. Antoine, A. L'Huillier, and M. Lewenstein, Attosecond Pulse Trains Using High-Order Harmonics, Phys. Rev. Lett. 77, 1234 (1996).

[3] P. M. Paul, E. S. Toma, P. Breger, G. Mullot, F. Augé, P. Balcou, H. G. Muller, and P. Agostini, Observation of a train of attosecond pulses from high harmonic generation, Science 292, 1689 (2001).

[4] P. Emma et al., First lasing and operation of an ångstromwavelength free-electron laser, Nat. Photonics 4, 641 (2010).

[5] C. Pellegrini, A. Marinelli, and S. Reiche, The physics of x-ray free-electron lasers, Rev. Mod. Phys. 88, 015006 (2016).

[6] P. Emma, K. Bane, M. Cornacchia, Z. Huang, H. Schlarb, G. Stupakov, and D. Walz, Femtosecond and Subfemtosecond X-Ray Pulses from a Self-Amplified 
Spontaneous-Emission-Based Free-Electron Laser, Phys. Rev. Lett. 92, 074801 (2004).

[7] Y. Ding, A. Brachmann, F. J. Decker, D. Dowell, P. Emma, J. Frisch, S. Gilevich, G. Hays, P. Hering, Z. Huang, R. Iverson, H. Loos, A. Miahnahri, H. D. Nuhn, D. Ratner, J. Turner, J. Welch, W. White, and J. Wu, Measurements and Simulations of Ultralow Emittance and Ultrashort Electron Beams in the Linac Coherent Light Source, Phys. Rev. Lett. 102, 254801 (2009).

[8] A. Marinelli, R. Coffee, S. Vetter, P. Hering, G. N. West, S. Gilevich, A. A. Lutman, S. Li, T. Maxwell, J. Galayda, A. Fry, and Z. Huang, Optical Shaping of X-Ray FreeElectron Lasers, Phys. Rev. Lett. 116, 254801 (2016).

[9] A. A. Lutman, T. J. Maxwell, J. P. MacArthur, M. W. Guetg, N. Berrah, R. N. Coffee, Y. Ding, Z. Huang, A. Marinelli, S. Moeller, and J. C. U. Zemella, Fresh-slice multicolour X-ray free-electron lasers, Nat. Photonics 10, 745 (2016).

[10] A. Marinelli, J. MacArthur, P. Emma, M. Guetg, C. Field, D. Kharakh, A. A. Lutman, Y. Ding, and Z. Huang, Experimental demonstration of a single-spike hard-X-ray free-electron laser starting from noise, Appl. Phys. Lett. 111, 151101 (2017).

[11] S. Huang, Y. Ding, Y. Feng, E. Hemsing, Z. Huang, J. Krzywinski, A. A. Lutman, A. Marinelli, T. J. Maxwell, and D. Zhu, Generating Single-Spike Hard X-Ray Pulses with Nonlinear Bunch Compression in Free-Electron Lasers, Phys. Rev. Lett. 119, 154801 (2017).

[12] E. Hemsing, G. Stupakov, D. Xiang, and A. Zholents, Beam by design: Laser manipulation of electrons in modern accelerators, Rev. Mod. Phys. 86, 897 (2014).

[13] A. A. Zholents, Method of an enhanced self-amplified spontaneous emission for x-ray free electron lasers, Phys. Rev. Accel. Beams 8, 040701 (2005).

[14] J. Duris et al., Tunable isolated attosecond X-ray pulses with gigawatt peak power from a free-electron laser, Nat. Photonics 14, 30 (2020).

[15] J. P. MacArthur, J. Duris, Z. Huang, and A. Marinelli, High power sub-femtosecond X-ray pulse study for the LCLS, in Proceedings of IPAC2017, Copenhagen, Denmark, 2017 (JACoW, Geneva, Switzerland, 2017).

[16] S. Serkez, G. Geloni, S. Tomin, G. Feng, E. V. Gryzlova, A. N. Grum-Grzhimailo, and M. Meyer, Overview of options for generating high-brightness attosecond x-ray pulses at free-electron lasers and applications at the European XFEL, J. Opt. 20, 024005 (2018).

[17] E. L. Saldin, E. A. Schneidmiller, and M. V. Yurkov, Selfamplified spontaneous emission FEL with energy-chirped electron beam and its application for generation of attosecond x-ray pulses, Phys. Rev. Accel. Beams 9, 050702 (2006).

[18] P. Baxevanis, J. Duris, Z. Huang, and A. Marinelli, Timedomain analysis of attosecond pulse generation in an X-ray free-electron laser, Phys. Rev. Accel. Beams 21, 110702 (2018).

[19] C. Emma, A. Lutman, M. W. Guetg, J. Krzywinski, A. Marinelli, J. Wu, and C. Pellegrini, Experimental demonstration of fresh bunch self-seeding in an X-ray free electron laser, Appl. Phys. Lett. 110, 154101 (2017).
[20] A. A. Lutman, M. W. Guetg, T. J. Maxwell, J. P. MacArthur, Y. Ding, C. Emma, J. Krzywinski, A. Marinelli, and Z. Huang, High-Power Femtosecond Soft $\mathrm{X}$ Rays from Fresh-Slice Multistage Free-Electron Lasers, Phys. Rev. Lett. 120, 264801 (2018).

[21] Takashi Tanaka, Proposal for a Pulse-Compression Scheme in X-Ray Free-Electron Lasers to Generate a Multiterawatt, Attosecond X-Ray Pulse, Phys. Rev. Lett. 110, 084801 (2013).

[22] E. Prat and S. Reiche, Simple Method to Generate Terawatt-Attosecond X-Ray Free-Electron-Laser Pulses, Phys. Rev. Lett. 114, 244801 (2015).

[23] T. Tanaka, Y. W. Parc, Y. Kida, R. Kinjo, C. H. Shim, I. S. Ko, B. Kim, D. E. Kim, and E. Prat, Using irregularly spaced current peaks to generate an isolated attosecond $\mathrm{X}$-ray pulse in free-electron lasers, J. Synchrotron Radiat. 23, 1273 (2016).

[24] A. M. Kondratenko and E. L. Saldin, Generation of coherent radiation by relativistic electron beam in an ondulator, Part. Accel. 10, 207 (1980).

[25] R. Bonifacio, C. Pellegrini, and L. M. Narducci, Collective instabilities and high-gain regime free electron laser, AIP Conf. Proc. 118, 236 (1984).

[26] R. Bonifacio, B. W. J. McNeil, and P. Pierini, Superradiance in the high-gain free-electron laser, Phys. Rev. A 40, 4467 (1989).

[27] L. Giannessi, P. Musumeci, and S. Spampinati, Nonlinear pulse evolution in seeded free-electron laser amplifiers and in free-electron laser cascades, J. Appl. Phys. 98, 043110 (2005).

[28] L. Giannessi and P. Musumeci, The free-electron laser harmonic cascade, New J. Phys. 8, 294 (2006).

[29] J. Arthur, W. Graves, M. Renner, J. Rosenzweig, G. Faigel, Z. Huang, and A. Ng, Linac coherent light source (LCLS) conceptual design, SLAC National Accelerator Laboratory Report No. SLAC-0593, 2002, https://www-ssrl.slac.stanford.edu/lcls/cdr/.

[30] M. Altarelli, R. Brinkmann, M. Chergui, W. Decking, B. Dobson, S. Dsterer, and J. Marangos, The European $\mathrm{X}$-ray free-electron laser technical design report, Report No. DESY-2006-097, 2007, https://www.osti.gov/ etdeweb/biblio/21285005.

[31] J. P. Duris, P. Musumeci, and R. K. Li, Inverse free electron laser accelerator for advanced light sources, Phys. Rev. Accel. Beams 15, 061301 (2012).

[32] U. Elu, T. Steinle, D. Snchez, L. Maidment, K. Zawilski, P. Schunemann, U. D. Zeitner, C. SimonBoisson, and J. Biegert, Table-top high-energy $7 \mu \mathrm{m}$ OPCPA and $260 \mathrm{~mJ}$ Ho:YLF pump laser, Opt. Lett. 44, 3194 (2019).

[33] S. Reiche, K. Goldhammer, and P. Musumeci, Recent upgrade to the free-electron laser code genesis 1.3 , in Proceedings of the 22nd Particle Accelerator Conference, PAC-2007, Albuquerque, NM (IEEE, New York, 2007), https://accelconf.web.cern.ch/AccelConf/p07/PAPERS/ TUPMS038.PDF.

[34] R. Bonifacio, L. D. S. Souza, P. Pierini, and N. Piovella, The superradiant regime of a FEL: Analytical and numerical results, Nucl. Instrum. Methods Phys. Res., Sect. A 296, 358 (1990). 
[35] N. M. Kroll, P. L. Morton, and M. N. Rosenbluth, Freeelectron lasers with variable parameter wigglers, IEEE J. Quantum Electron. 17, 1436 (1981).

[36] Z. Zhang, J. Duris, J. P. MacArthur, Z. Huang, and A. Marinelli, Double chirp-taper x-ray free-electron laser for attosecond pump-probe experiments, Phys. Rev. Accel. Beams 22, 050701 (2019).

[37] J. M. Glownia, J. Cryan, J. Andreasson, A. Belkacem, N. Berrah, C. I. Blaga, C. Bostedt, J. Bozek, L. F. DiMauro, L. Fang, and J. Frisch, Time-resolved pumpprobe experiments at the LCLS, Opt. Express 18, 17620 (2010).
[38] J. P. MacArthur, J. Duris, Z. Zhang, Z. Huang, and A. Marinelli, Self-modulation of a relativistic electron beam in a wiggler, in Proceedings of the 9th International Particle Accelerator Conference (IPAC2018), Vancouver, BC, Canada, 2018 (JACoW Publishing, Geneva, Switzerland, 2018).

[39] J. P. MacArthur, J. Duris, Z. Zhang, A. Lutman, A. Zholents, X. Xu, Z. Huang, and A. Marinelli, Phase-Stable Self-Modulation of an Electron Beam in a Magnetic Wiggler, Phys. Rev. Lett. 123, 214801 (2019). 